\title{
Proapoptotic protein Bim attenuates estrogen-enhanced survival in lymphangioleiomyomatosis
}

Chenggang Li, ${ }^{1} \mathrm{Na}$ Li, ${ }^{1,2}$ Xiaolei Liu, ${ }^{1}$ Erik Y. Zhang, ${ }^{1}$ Yang Sun, ${ }^{1}$ Kouhei Masuda, ${ }^{1}$ Jing Li, ${ }^{3}$ Julia Sun, ${ }^{1}$ Tasha Morrison, ${ }^{4}$ Xiangke Li, ${ }^{1,2}$ Yuanguang Chen, ${ }^{1,5}$ Jiang Wang, ${ }^{6}$ Nagla A. Karim, ${ }^{7}$ Yi Zhang, ${ }^{8}$ John Blenis, ${ }^{3,9}$ Mauricio J. Reginato, ${ }^{10}$ Elizabeth P. Henske, ${ }^{11}$ and Jane J. Yu ${ }^{1,2}$

'University of Cincinnati College of Medicine, Department of Internal Medicine, Pulmonary, Critical Care and Sleep Medicine, Cincinnati, Ohio, USA. ${ }^{2}$ The First Affiliated Hospital of Zhengzhou University, Department of Oncology, Zhengzhou, Henan, China. ${ }^{~}$ Harvard Medical School, Department of Cell Biology, Boston, Massachusetts, USA. ${ }^{4}$ Boston University School of Medicine, Department Molecular and Translational Medicine, Boston, Massachusetts, USA. ${ }^{5}$ The First Affiliated Hospital of Guangzhou Medical University, Department of Gastrointestinal Surgery, Guangzhou, China. ${ }^{6}$ University of Cincinnati College of Medicine, Department of Pathology and Lab Medicine, Cincinnati, OH, USA. ־University of Cincinnati College of Medicine, Department of Internal Medicine, Division of Hematology and Oncology, Cincinnati, Ohio, USA. ${ }^{8}$ The First Affiliated Hospital of Zhengzhou University, Biotherapy Center and Department of

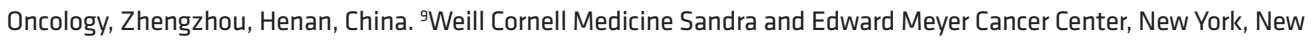
York, USA. ${ }^{10}$ Drexel University College of Medicine, Department of Biochemistry and Molecular Biology, Philadelphia, Pennsylvania, USA. "Brigham and Women's Hospital-Harvard Medical School, Boston, Massachusetts, USA.

Lymphangioleiomyomatosis (LAM) is a progressive lung disease that primarily affects young women. Genetic evidence suggests that LAM cells bearing TSC2 mutations migrate to the lungs, proliferate, and cause cystic remodeling. The female predominance indicates that estrogen plays a critical role in LAM pathogenesis, and we have proposed that estrogen promotes LAM cell metastasis by inhibition of anoikis. We report here that estrogen increased LAM patient-derived cells' resistance to anoikis in vitro, accompanied by decreased accumulation of the proapoptotic protein Bim, an activator of anoikis. The resistance to anoikis was reversed by the proteasome inhibitor, bortezomib. Treatment of LAM patient-derived cells with estrogen plus bortezomib promoted anoikis compared with estrogen alone. Depletion of Bim by siRNA in TSC2-deficient cells resulted in anoikis resistance. Treatment of mice with bortezomib reduced estrogen-promoted lung colonization of TSC2-deficient cells. Importantly, molecular depletion of Bim by siRNA in Tsc2deficient cells increased lung colonization in a mouse model. Collectively, these data indicate that Bim plays a key role in estrogen-enhanced survival of LAM patient-derived cells under detached conditions that occur with dissemination. Thus, targeting Bim may be a plausible future treatment strategy in patients with LAM.

Conflict of interest: The authors declare that no conflict of interest exists.

Submitted: January 20, 2016 Accepted: October 10, 2016 Published: November 17, 2016

Reference information: JCI Insight. 2016;1(19):e86629. doi:10.1172/jici.insight.86629.

\section{Introduction}

Lymphangioleiomyomatosis (LAM) is a devastating disease affecting young women. The proposed pathogenesis of LAM holds that histologically benign-appearing smooth muscle cells arise from an unknown source and metastasize to the lungs, where they promote destructive cystic remodeling. About $30 \%-40 \%$ of women with tuberous sclerosis complex (TSC), a genetic disorder caused by TSC1 and TSC2 gene defects, have radiographic evidence of $\operatorname{LAM}(1,2)$. A Mayo Clinic study of a series of TSC patients reported that LAM was one of the leading causes of death in women with TSC (3). LAM also occurs in a sporadic form (S-LAM) in women who do not have TSC. In those patients, somatic TSC2 mutations have been reported in lesional cells from the lung, kidney, and lymphatics, but not in normal tissues from those organs or in circulating myeloid cells (4). Multiple preclinical studies demonstrated the effectiveness of rapamycin, an mTORC1 inhibitor, in animal models of TSC (5), which led to rapid clinical translation, and demonstration that rapamycin has clinical benefit in patients with LAM (6). In patients with end-stage LAM, lung transplantation is offered as a last resort, although recurrence of LAM has been reported in the donor allograft (7). 
The reasons that LAM exclusively affects women remain unclear. The remarkable female predominance of LAM suggests that female hormones, including estrogen, may contribute to disease pathogenesis. Both LAM cells and angiomyolipoma cells express estrogen receptor $\alpha$, estrogen receptor $\beta$, and the progesterone receptor (8). We have previously discovered that estrogen promotes the survival and lung colonization of intravenously injected Tsc2-deficient rat-uterine leiomyoma-derived ELT3 cells in our preclinical mouse model of LAM (9). In a similar xenograft tumor model, estrogen strongly enhanced the pulmonary metastasis of ELT3 cells, associated with an increase in MEK1/2-Erk1/2 signaling in circulating tumor cells. Collectively, our data indicate that estrogen plays a key role in promoting the survival of disseminated TSC2-deficient LAM-derived cells during disease progression (9), although the precise mechanisms involved have remained elusive.

LAM has been described as a destructive, low-grade metastasizing neoplasm (10). Cells carrying TSC2 mutations have been identified in body fluids including blood, chylous effusions, and urine from women with LAM (11). Tumor cells become metastatic by dissociation from primary sites, survival in the vascular system, and proliferation in distal organs. Cells normally undergo anoikis (anchorage-dependent programmed cell death) after losing contact with extracellular matrix or neighboring cells. As a neoplastic strategy, tumor cells acquire resistance to anoikis to allow survival after detachment from the primary site and dissemination via lymphatic or vascular channels. Tumor cells can acquire resistance to anoikis through genetic mutations that lead to inactivation of the death receptor pathway of caspase activation or overexpression of antiapoptotic proteins, including Bcl-2 family members that contain the Bcl-2 homology (BH) domain 3 (11-13). The Bcl-2-interacting mediator of cell death (Bim), a BH3-only protein, is a critical activator of anoikis. In healthy cells, Bim is inactivated by its interaction with the cytoskeleton (14-18). Upon activation by apoptotic stimuli, Bim is phosphorylated by Erk1/2. Phosphorylation of Bim also promotes rapid proteasome-mediated degradation that facilitates cell survival (19-21).

In this study, we tested the hypothesis that Bim is a critical mediator of estrogen-supported survival of TSC2-deficient LAM patient-derived cells. We report here that estrogen decreases Bim accumulation and induces resistance of LAM patient-derived cells to anoikis. Furthermore, depletion of Bim by small interfering RNA (siRNA) prevents anoikis in LAM patient-derived cells. Bortezomib treatment restores susceptibility to anoikis in estrogen-treated LAM cells by preventing Bim degradation. In vivo, pharmacological suppression of proteasome activity using bortezomib increases Bim accumulation and reduces estrogen-promoted lung colonization of Tsc2-deficient cells, and molecular depletion of Bim by siRNA resulted in enhanced survival of Tsc2-deficient cells in a mouse model of LAM.

\section{Results}

Estrogen-promoted resistance of TSC2-deficient LAM patient-derived cells to anoikis is associated with Erk1/2 and Akt activation in vitro. Previously, we showed that estrogen $\left(\mathrm{E}_{2}\right)$ induced the survival of Tsc2-deficient Eker leiomyoma-derived (ELT3) cells in detachment conditions (9). To determine the mechanism responsible for $\mathrm{E}_{2}$-enhanced resistance of ELT3 cells to anoikis, we analyzed the effects of $\mathrm{E}_{2}$ and Bim on anoikis. $\mathrm{E}_{2}$ treatment decreased the population of late apoptotic (annexin $\mathrm{V}^{+}$propidium iodide ${ }^{+}\left[\mathrm{PI}^{+}\right]$) cells compared with that of untreated cells $(35.2 \pm 1.0$ vs. $20.3 \pm 0.7)$ in detachment conditions, as analyzed by flow cytometry (Figure 1A). Similarly, $\mathrm{E}_{2}$ treatment decreased the population of late apoptotic (annexin $\mathrm{V}^{+} \mathrm{PI}^{+}$) cells compared with untreated LAM patient-derived cells (621-101 cells) (22) (45.6 \pm 0.1 vs. 4.2 \pm 0.3 ) (Figure 1A), suggesting that TSC2-deficient cells were protected from anoikis by $\mathrm{E}_{2}$ treatment in detachment conditions. $\mathrm{E}_{2}$ treatment also decreased the level of Bim protein in detachment conditions by $40 \%$ and 60\%, within 60 minutes and 120 minutes, respectively (Figure 1B), which was inversely correlated with Bim stability.

Mutation of TSC2 leads to hyperactivation of mTORC1 and dysregulation of PI3K-Akt via feedback inhibition mechanisms (23). The expression of Bim is regulated both transcriptionally and posttranscriptionally by PI3K/Akt and Erk1/2 signaling networks (24). We next assessed the effect of $\mathrm{E}_{2}$ on the activation of these signaling molecules in TSC2-deficient cells grown in detachment conditions. $\mathrm{E}_{2}$ treatment resulted in Erk1/2 phosphorylation within 5 minutes that continued up to 30 minutes after treatment, and Akt phosphorylation (Ser473) within 30 minutes that lasted up to 120 minutes after treatment in detached ELT3 cells (Figure 1B), indicating a correlation between $\mathrm{E}_{2}$-induced Bim downregulation and reactivation of Akt and Erk1/2 in Tsc2-deficient cells grown in detachment. To determine whether Bim expression is related to the biochemical features of TSC2-deficient cells, we compared the protein levels of Bim and S6 activation in detached ELT3 
A

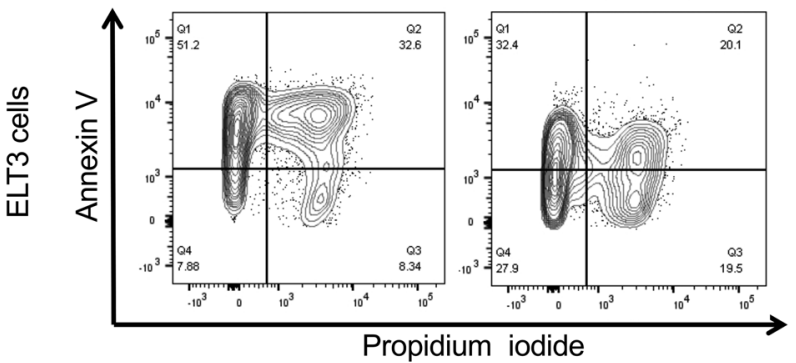

Propidium iodide

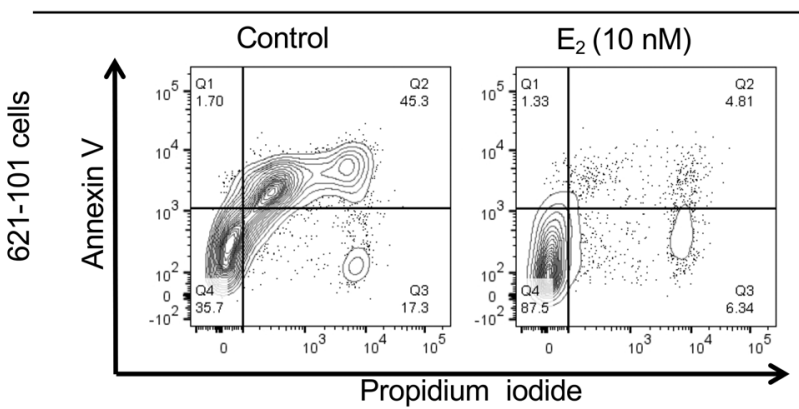

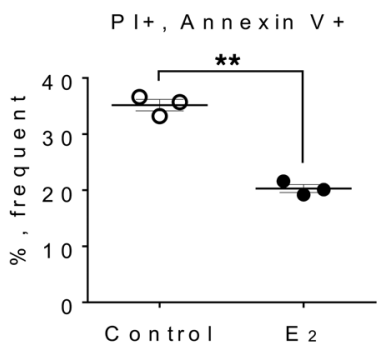

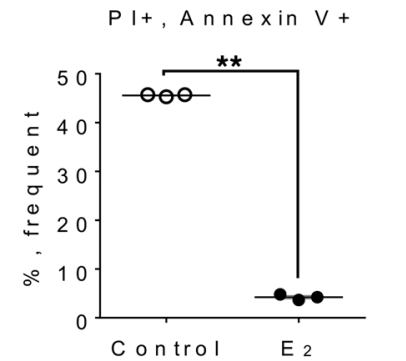

B

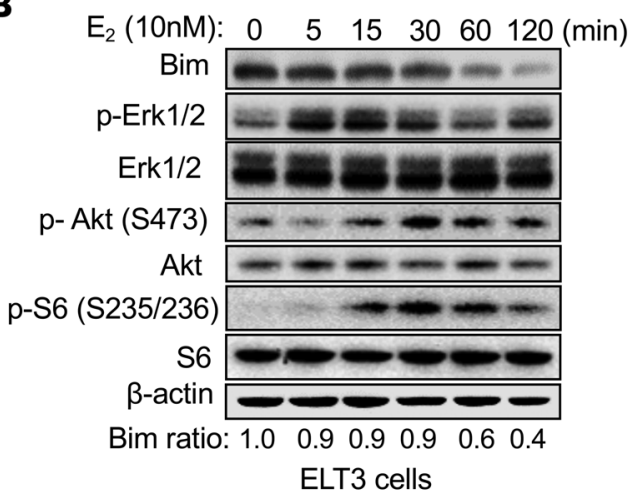

C

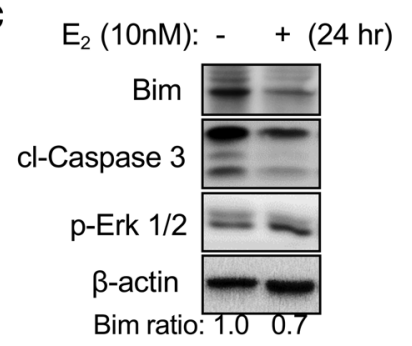

D

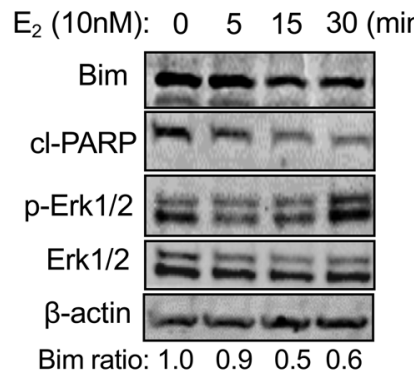

621-101 cells
Figure 1. Estrogen decreases Bim expression in detached ELT3 cells and 621-101 cells. (A) Tsc2-deficient rat uterine leiomyoma-derived (ELT3) cells (upper panels) and TSC2-deficient LAM patient-derived cells (621-101 cells) (lower panels) were seeded in poly-HEMA-coated plates, and treated with $10 \mathrm{nM}$ $17-\beta$-estradiol $\left(E_{2}\right)$ for 24 hours. Cells were stained with a BD Annexin V: FITC Apoptosis Detection Kit I. Anoikis was determined by flow cytometry (BD FACSCanto II). Annexin $\mathrm{V}^{+} \mathrm{Pl}^{+}$indicated cells undergoing anoikis $(n=3)$. (B) Immunoblotting analysis of Bim levels in ELT3 cells treated with $E_{2}$ for indicated periods. (C) 621-101 cells were treated with $10 \mathrm{nM} \mathrm{E}_{2}$ or vehicle control for 24 hours in detachment conditions. Cleaved caspase 3 was used as an additional cell death marker. (D) 621-101 cells were treated with $10 \mathrm{nME}_{2}$ for 5,15 , and 30 minutes in detachment conditions. Immunoblotting analyses of Bim, phospho-Erk1/2, Erk1/2, and cleaved PARP were performed. In B-D, $\beta$-actin was used as a loading control; Bim levels were quantified using densitometry, and normalized to $\beta$-actin. Results are representative of 3 experiments. Statistical analysis performed using an unpaired, 2-tailed Student's $t$ test. ${ }^{* *} P<0.01$ was considered significant.

cells. $\mathrm{E}_{2}$ treatment decreased the levels of Bim protein by $40 \%$ and $60 \%$, at 60 minutes and 120 minutes, respectively. Interestingly, S6 phosphorylation was undetectable within the first 5 minutes, and gradually increased between 15 and 60 minutes of $E_{2}$ stimulation

(Figure 1B), representing an inverse correlation between Tsc2 deficiency and Bim expression, in agreement with an earlier report that loss of Tsc2 leads to anoikis resistance and anchorage-independent growth (25). TSC2-deficient cells exhibit hyperactivation of mTORC1, thus leading to constitutive phosphorylation of S6. We found that S6 phosphorylation was undetectable in Tsc2-deficient ELT3 cells grown in suspension (Figure 1B), indicative of detachment-induced suppression of $\mathrm{mTORC} 1$ signaling. Furthermore, $\mathrm{E}_{2}$ treatment of 621-101 cells for 24 hours decreased the protein levels of Bim by $39 \%$ relative to control (Figure 1C), and correlated with the increased phosphorylation of Erk1/2 in detachment conditions. Importantly, decreased anoikis was associated with decreased levels of cleaved caspase 3 in $\mathrm{E}_{2}$-treated 621-101 cells compared with control cells (Figure 1C). Next, we examined the rapid effect of $\mathrm{E}_{2}$ on Bim levels in detached 621-101 cells. $\mathrm{E}_{2}$ treatment decreased the levels of Bim protein by $50 \%$ and $40 \%$ within 15 minutes and 30 minutes, respectively (Figure 1D). Importantly, the $\mathrm{E}_{2}$-promoted reduction of Bim was associated with Erk1/2 activation and PARP cleavage, suggesting that $\mathrm{E}_{2}$ protects $\mathrm{LAM}$ patient-derived cells from detachment-promoted apoptosis.

Bim depletion promotes anoikis resistance of TSC2-deficient cells. To determine whether Bim is a key mediator of detachment-induced death in Tsc2-deficient ELT3 cells, we knocked down Bim using 2 independent 
A
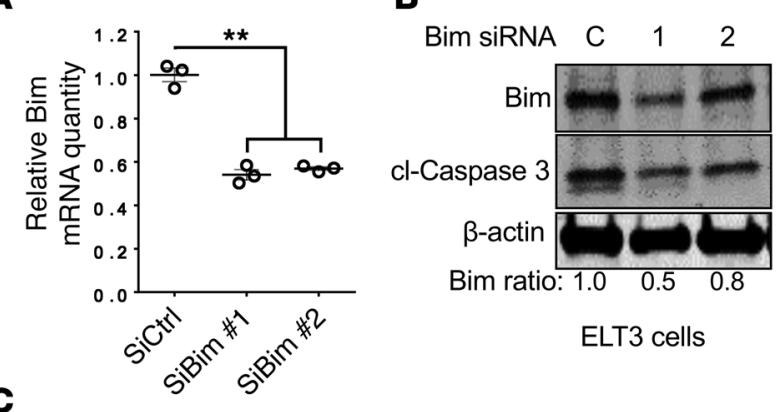

ELT3 cells
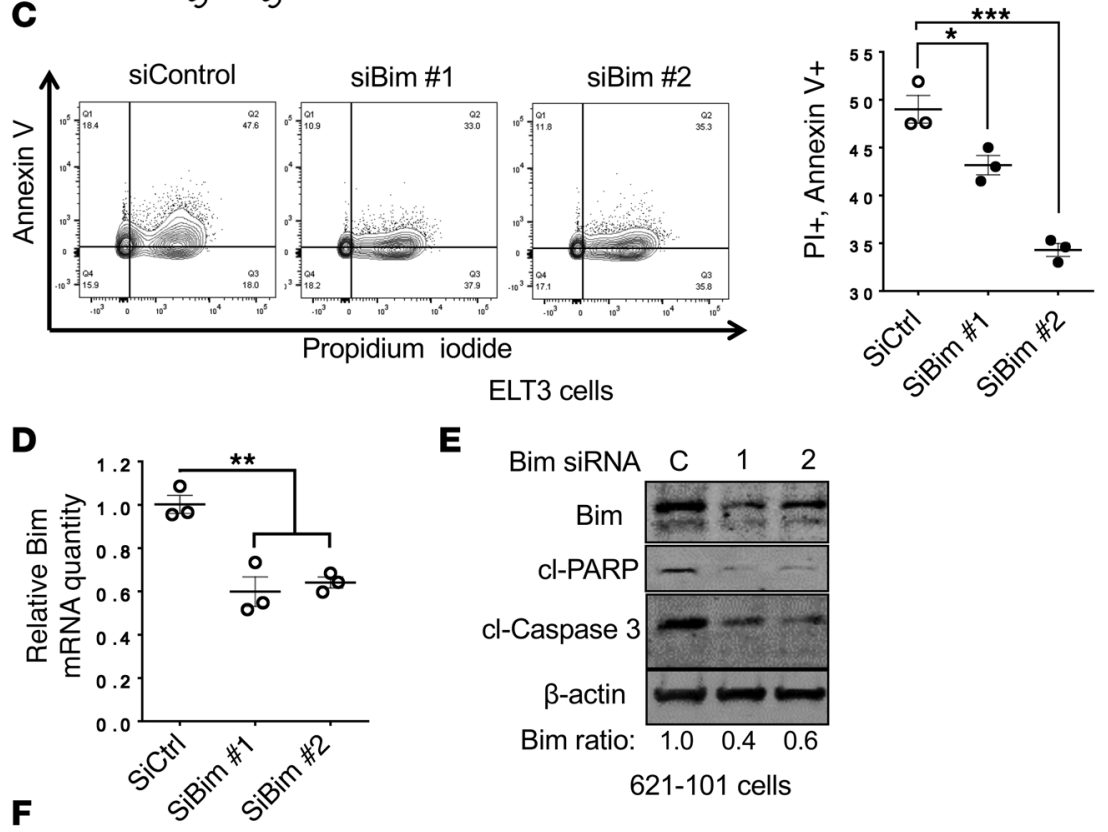

E

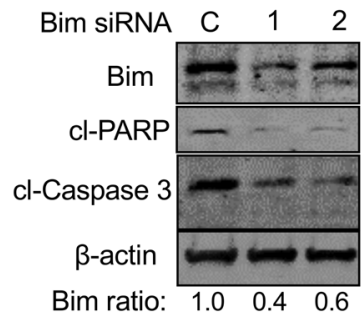

621-101 cells

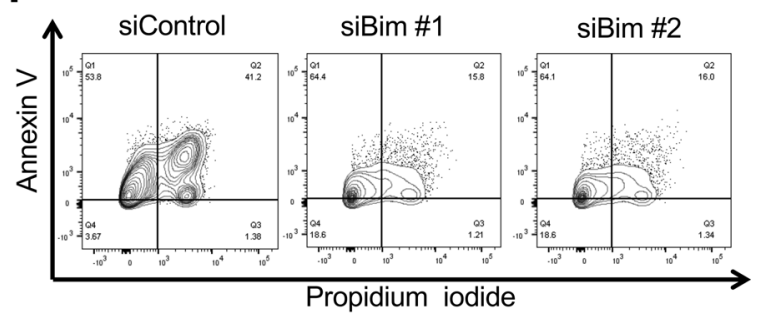

621-101 cells

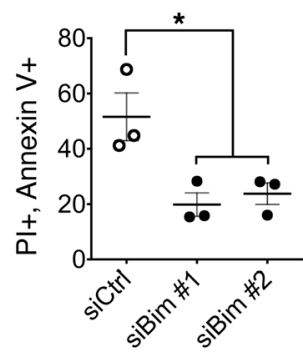

Figure 2. Bim is the key mediator of anoikis. (A) ELT3 cells were transfected with 2 independent rat Bim siRNAs or control siRNA for 48 hours. Bim transcript levels were measured using real-time RT-PCR ( $n$ = 3). (B) ELT3 cells were transfected with 2 independent rat Bim siRNAs or control siRNA for 48 hours, followed by 24 -hour culture in poly-HEMA-coated plates. Levels of Bim, cleaved caspase 3 , and cleaved PARP (rat) were analyzed by immunoblotting; Bim levels were quantified using densitometry, and normalized to $\beta$-actin. (C) ELT3 cells transfected with 2 independent rat Bim siRNAs were stained with a BD Annexin V: FITC Apoptosis Detection Kit I. Anoikis was determined by flow cytometry (BD FACSCanto II). Annexin $\mathrm{V}^{+} \mathrm{PI}^{+}$indicated cells undergoing anoikis $(n=3)$. (D) 621-101 cells were transfected with 2 independent human Bim siRNAs or control siRNA for 48 hours. Bim transcript levels were measured using real-time RT-PCR $(n=3)$. (E) 621-101 cells were transfected with 2 independent human Bim siRNAs or control siRNA for 48 hours, followed by a 24-hour culture in poly-HEMA-coated plates. Levels of Bim, cleaved caspase 3 , and cleaved PARP (human) were analyzed by immunoblotting; Bim levels were quantified using densitometry, and normalized to $\beta$-actin. (F) 621-101 cells transfected with 2 independent human Bim siRNAs were stained with a BD Annexin V: FITC Apoptosis Detection Kit I. Anoikis was determined by flow cytometry (BD FACSCanto II). Annexin $\mathrm{V}^{+} \mathrm{Pl}^{+}$indicated cells undergoing anoikis $(n=3)$. Results are representative of 3 experiments. Statistical analysis performed using a 1-way ANOVA test (Dunnett's multiple comparisons test comparing Bim siRNA-1, Bim siRNA-2 with siControl). ${ }^{*} P<0.05$, ${ }^{* *} P<0.01$, or ${ }^{* *} P<0.005$ was considered significant.

Bim siRNAs. We found that ELT3 cells transfected with these 2 rat Bim siRNAs exhibited $50 \%$ and $52 \%$ reduction of the transcript levels of Bim measured by real-time RT-PCR (Figure 2A), and 50\% and 20\% decrease of the protein levels of Bim, based on densitometry of immunoblots using an anti-Bim antibody, relative to control siRNA treatment (Figure 2B). Next, we compared the levels of cleaved caspase 3 as a marker of cell death. ELT3 cells were transfected with the 2 Bim siRNAs for 24 hours in adherent conditions, collected, and cultured in poly(2-hydroxyethyl methacrylate) (poly-HEMA)-coated plates for 24 hours, and then analyzed by immunoblotting. The results show that Bim siRNA-transfected ELT3 cells had lower levels of cleaved caspase 3 compared with cells transfected with control siRNA (Figure 2B), suggesting that Bim is a critical mediator of detachment-triggered death of ELT3 cells. Furthermore, we used flow cytometry to assess the effect of Bim depletion on the survival of ELT3 cells in detachment conditions. The percentage of ELT3 cells in late apoptosis (annexin $\mathrm{V}^{+} \mathrm{PI}^{+}$) was decreased from $49 \%$ to $43 \%$, or from $49 \%$ to $33 \%$, after 2 independent Bim knockdowns (Figure 2C), further supporting Bim as a critical mediator of detachment-promoted death of Tsc2-deficient cells.

Next, we depleted Bim using 2 independent human Bim siRNAs in 621-101 cells. Forty-eight hours after siRNA transfection, the transcript levels of Bim were reduced by $41 \%$ and $38 \%$ (Figure 2D), and protein 
A

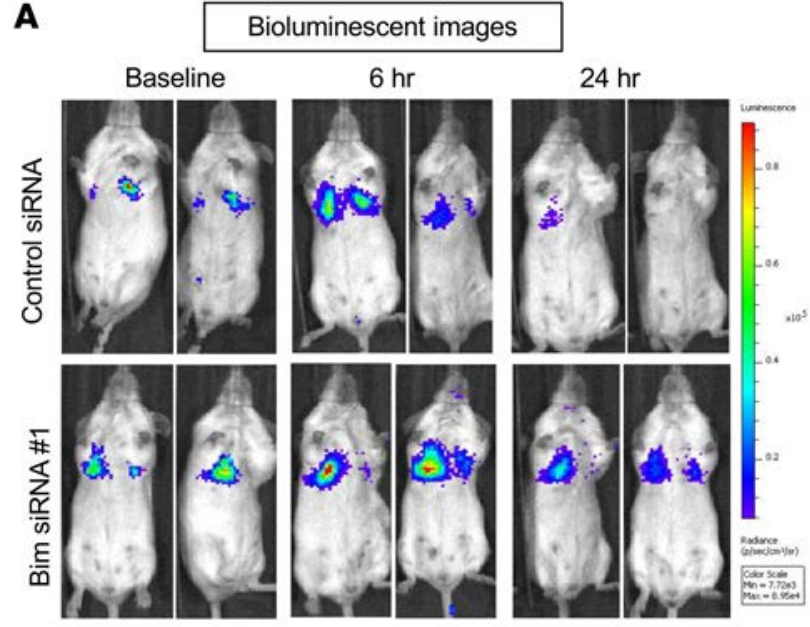

B

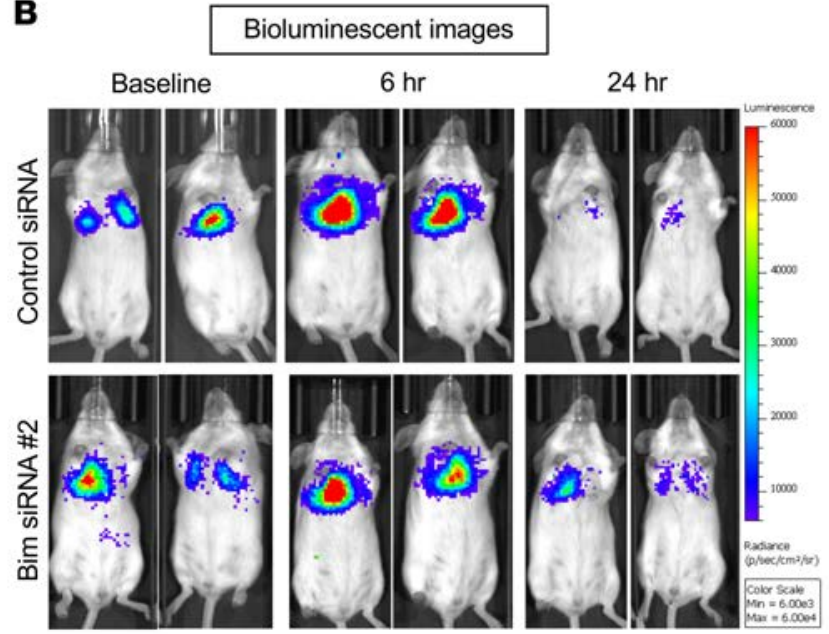

C Baseline Photon Flux

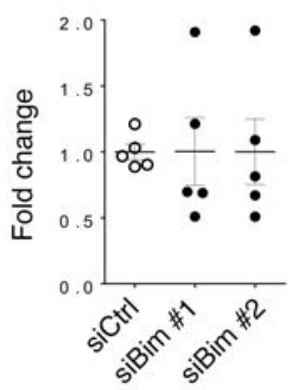

$6 \mathrm{hr}$ Photon Flux

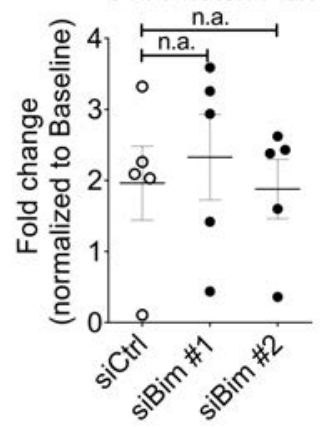

24hr Photon Flux

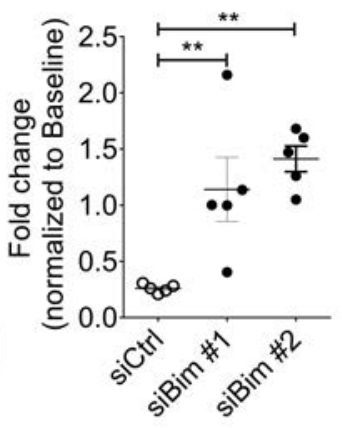

Figure 3. Bim depletion increases lung accumulation of ELT3 cells in vivo. (A and B) Luciferase-expressing ELT3 (ERL4) cells transfected with 2 independent Bim siRNAs (both $n=5)$ or control siRNA $(n=5)$ for 24 hours were injected into female SCID mice intravenously; the bioluminescence images were taken at 0,6 , and 24 hours after cell injection. (C) Bioluminescence intensity was recorded and quantified. Scatter plots show the levels of lung colonization of ERL 4 cells as the mean of bioluminescence intensity from 5 mice per group. Statistical analysis performed using a 1-way ANOVA test (Dunnett's multiple comparisons test comparing Bim siRNA 1 , Bim siRNA 2 with siControl). ${ }^{* *} P<0.01$ was considered significant.

levels were decreased by $60 \%$ and $40 \%$, relative to control siRNA treatment (Figure $2 \mathrm{E})$. Levels of cleaved PARP and cleaved caspase 3 were markedly decreased in 621 101 cells transfected with the 2 Bim siRNAs compared with control siRNA treatment (Figure 2E). Moreover, the percentage of 621-101 cells in late apoptosis (annexin $\mathrm{V}^{+} \mathrm{PI}^{+}$) was decreased from $52 \%$ to $20 \%$, or from $52 \%$ to $24 \%$, after 2 independent Bim knockdowns (Figure 2F). Collectively, our findings demonstrate that Bim is a critical regulator of detachment-induced apoptosis of TSC2-deficient cells.

Bim mediates $E_{2}$-promoted cell survival and lung colonization of Tsc2-deficient ELT3 cells in vivo. To investigate the impact of Bim-mediated anoikis on tumor cell survival in a preclinical model of LAM, we intravenously injected female SCID mice with ELT3 luciferaseexpressing (ERL4) cells transfected with rat Bim siRNA (number 1) or control siRNA. At 6 hours after cell injection, Bim-depleted cells colonized the lungs more extensively than control siRNA-transfected cells, as indicated by bioluminescence intensity (Figure 3A), although this difference was not statistically significant. Importantly, at 24 hours after cell injection, the control siRNA-transfected cells were barely detectable in the lungs of SCID mice, whereas the Bim-knockdown cells showed a strong and persistent bioluminescence signal. To determine the specificity of Bim depletion in lung colonization, we transfected ERL4 cells with a second Bim siRNA (number 2) or control siRNA, and then injected these cells intravenously into female SCID mice. At 6 hours after cell injection, all mice exhibited enhanced bioluminescence intensity. Importantly, at 24 hours after cell injection, the control siRNA-transfected cells were barely detectable in the lungs of SCID mice, whereas the Bim-knockdown cells showed markedly greater bioluminescence signal relative to the controls (Figure 3B). Quantification of the bioluminescence intensity in mouse lungs showed that ERL4 cells treated with the 2 independent Bim siRNAs exhibited a 5-fold and 6-fold increase in lung colonization relative to control siRNA treatments (Figure 3C). Collectively, these data further support the notion that Bim is a critical regulator of the survival of TSC2-deficient cells in vivo.

$E_{2}$ activates Erk1/2 and increases anoikis resistance of ELT3 and LAM patient-derived cells via Bim degradation in vitro. We have found that Erk1/2 is highly activated by $\mathrm{E}_{2}$ treatment in ELT3 cells and 621-101 cells in vitro (Figure 1) and that $\mathrm{E}_{2}$ treatment leads to increased lung colonization of ELT3-luciferase cells in vivo (7). To investigate the molecular mechanisms of these effects, we examined signaling events underlying the reduced levels of Bim in $\mathrm{E}_{2}$-treated ELT3 cells and 621-101 cells. Flow cytometry analyses showed that $\mathrm{E}_{2}$ 


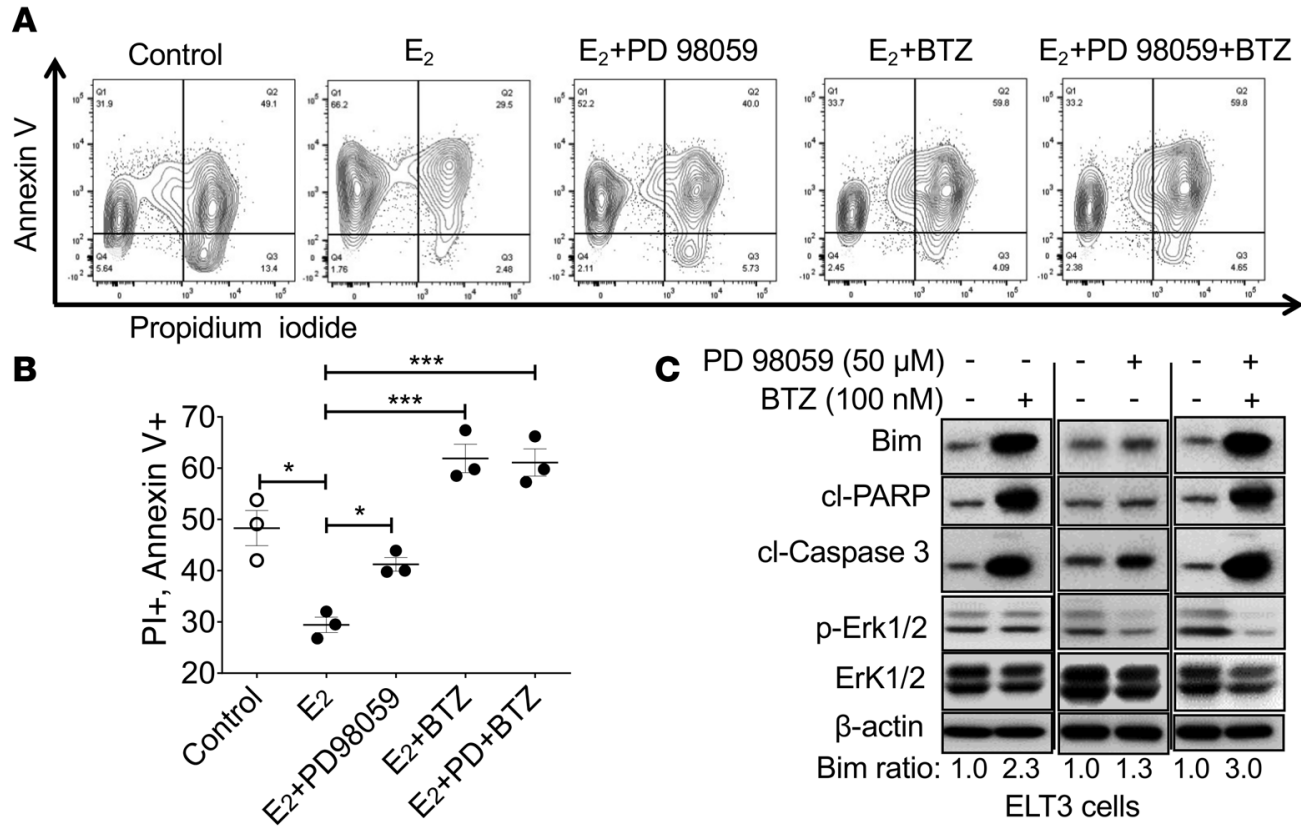

D 601-101 cells
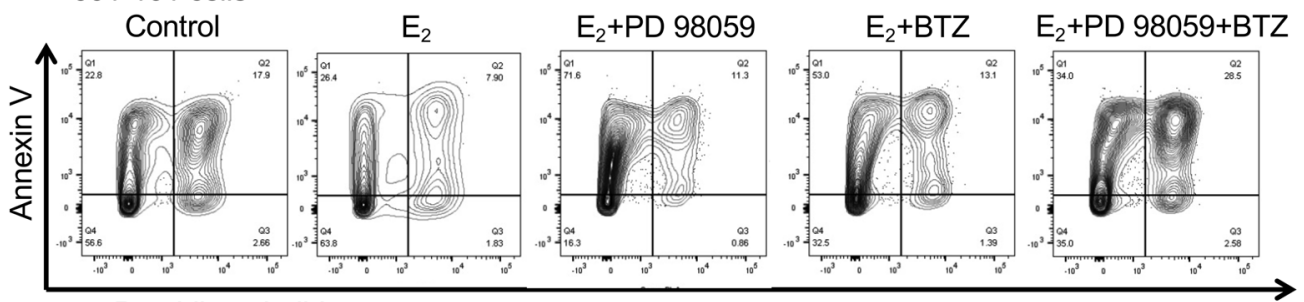

E

Propidium iodide

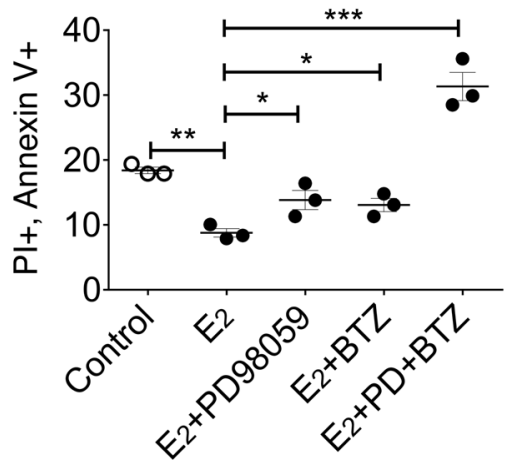

$\mathbf{F}$

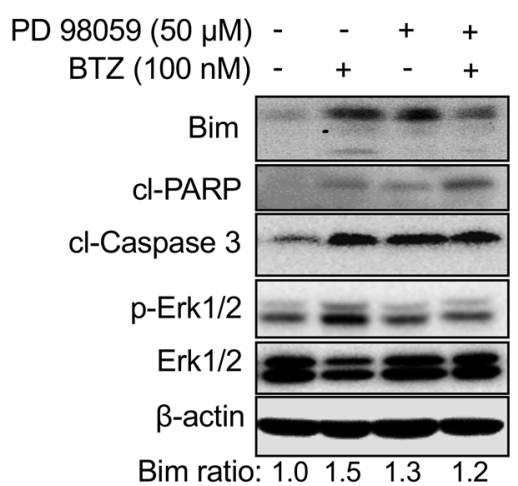

601-101 cells

Figure 4. Erk1/2 and proteasome inhibition decreases estrogen-promoted cell survival and restores Bim levels in LAM cells. (A) ELT3 cells were seeded in poly-HEMA-coated plates, pretreated with or without $50 \mu \mathrm{M}$ PD98059, a selective Erk1/2 inhibitor, or 100 nM proteasome inhibitor bortezomib (BTZ), or both PD98059 and BTZ for 15 minutes. The treatments were continued for an additional 24 hours in the presence of $10 \mathrm{nM}$ estrogen $\left(E_{2}\right)$ or vehicle control. Cell death was analyzed by flow cytometry $(n=3)$. (B) The percentage of late apoptotic (annexin $\left.\mathrm{V}^{+} \mathrm{Pl}^{+}\right)$cells was determined $(n=3)$. (C) Immunoblotting analysis shows levels of phospho-Erk1/2, Erk1/2, Bim, and cell death markers cleaved caspase 3 and cleaved PARP in detached ELT3

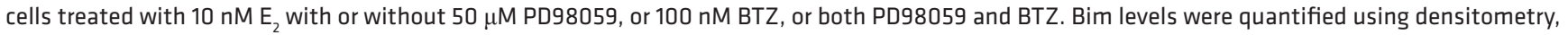
and normalized to $\beta$-actin. The lanes were run on the same gel, but were noncontiguous. (D) 621-101 cells were seeded in poly-HEMA-coated plates,

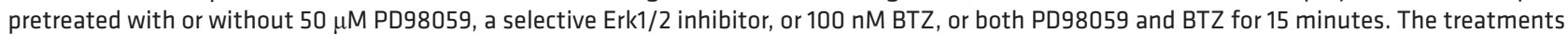
were continued for an additional 24 hours in the presence of $E_{2}(10 \mathrm{nM})$ or vehicle control. Cell death was analyzed by flow cytometry $(n=3)$. $(E)$ The percentage of late apoptotic (annexin $\mathrm{V}^{+} \mathrm{Pl}^{+}$) cells was determined $(n=3)$. (F) Immunoblotting analysis shows levels of phospho-Erk1/2, Erk1/2, Bim and cell death markers cleaved caspase 3 and cleaved PARP in detached 621-101 cells treated with $10 \mathrm{nM} \mathrm{E}$, with or without $50 \mu \mathrm{M}$ PD98059, or 100 nM BTZ, or both PD98059 and BTZ. Bim levels were quantified using densitometry, and normalized to $\beta$-actin (obtained from replicate samples run in parallel). Results are representative of 3 experiments. Statistical analysis performed using a 1-way ANOVA test (Tukey's multiple comparisons test multiple pairwise comparisons between different groups). ${ }^{*} P<0.05$, ${ }^{* *} P<0.01$, or ${ }^{* *} P<0.005$ was considered significant. 
A

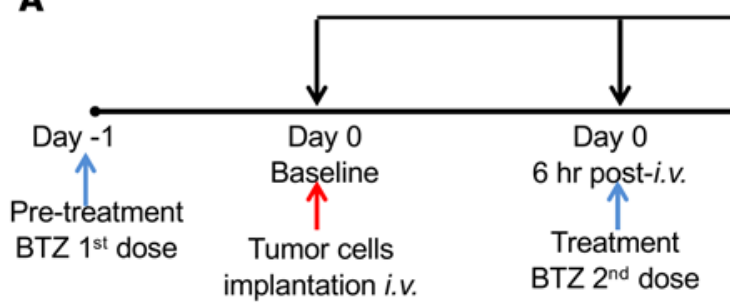

B

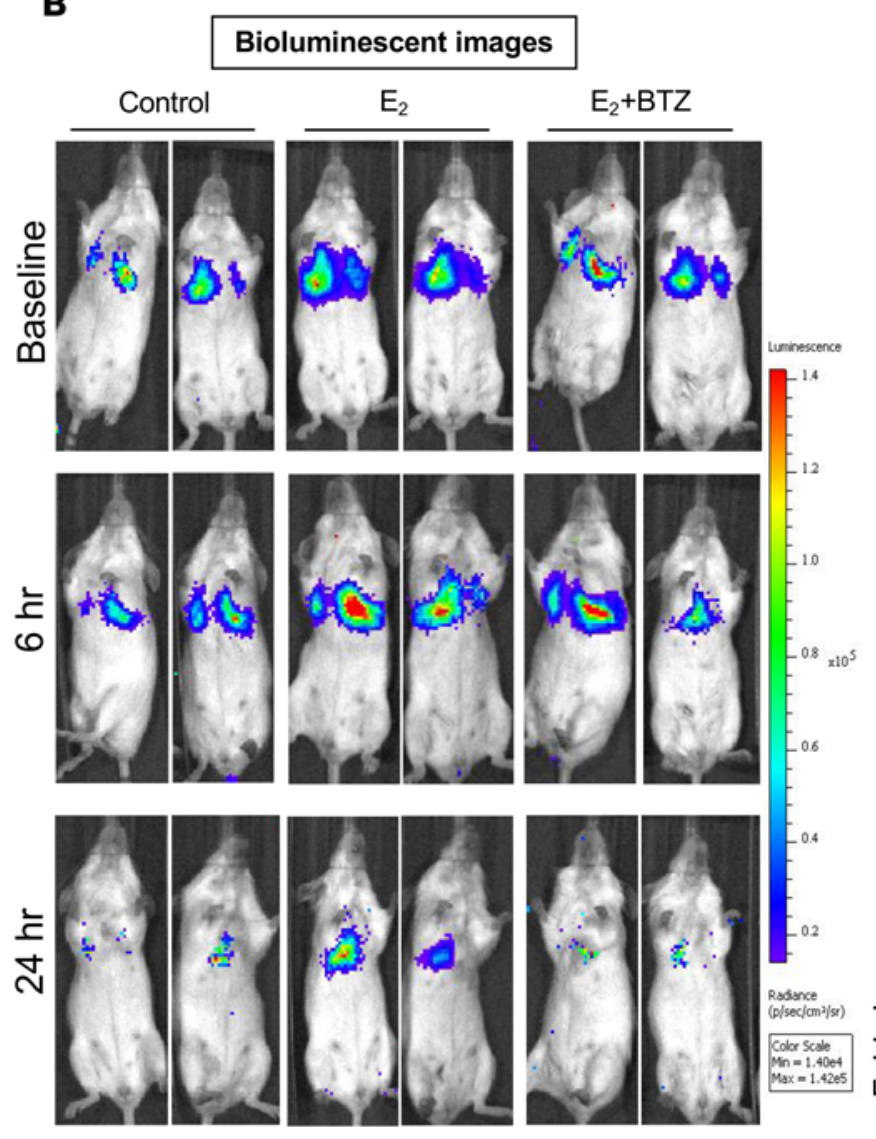

Figure 5. The proteasome inhibitor bortezomib blocks lung colonization of ELT3 cells in vivo. (A) Simplified study flow. Female ovariectomized SCID mice receiving vehicle control ( $n$ $=5$ ) or estrogen $\left(E_{2}\right)$ in drinking water were treated with bortezomib (BTZ; $1 \mathrm{mg} / \mathrm{kg}$, dissolved in $100 \mu \mathrm{l}$ PBS, i.p., $n=5$ ) or control (sterilized PBS, $n=5$ ) 24 hours in advance of cell injection and 6 hours after cell inoculation. Luciferase-expressing ELT3 (ERL4) cells were injected in female SCID mice intravenously; the baseline bioluminescence images were captured immediately, and 6 and 24 hours after cell injection. A second dose of BTZ or control was administered 6 hours after cell injection. (B) Bioluminescence imaging was performed at the indicated times. (C) Bioluminescence intensity (total photon flux) was recorded and quantified. Data are the mean bioluminescence intensities from 5 mice per group. Statistical analysis performed using a 1-way ANOVA test (Tukey's multiple comparisons test multiple pair-wise comparisons between different groups). ${ }^{*} P<0.05$ or ${ }^{* *} P<0.01$ was considered significant.

treatment decreased the population of detached cells in late apoptosis (annexin $\mathrm{V}^{+} \mathrm{PI}^{+}$) by $40 \%$ (Figure 4, $\mathrm{A}$ and $\mathrm{B}$ ). We also found that the $\mathrm{E}_{2}$-mediated decrease in the population of cells in apoptosis was partially attenuated by $\sim 45 \%$ upon treatment with the Erk1/2 inhibitor PD98059 (Figure 4, A and B). Similarly, PD98059 treatment restored the protein levels of Bim in $\mathrm{E}_{2}$-treated ELT3 cells (Figure 4C). Importantly, cells treated with $\mathrm{E}_{2}$ plus PD98059 exhibited higher levels of cleaved caspase 3 compared with $\mathrm{E}_{2}$ treatment alone (Figure 4C). Similar findings were observed in detached 621-101 cells (Figure 4, D-F). Together, these data support a role for Bim in mediating $\mathrm{E}_{2}$-protected anoikis via Erk1/2 in TSC2-deficient ELT3 and 621-101 cells.

Bortezomib restores Bim-mediated anoikis of ELT3 and LAM patient-derived cells in vitro. The results above indicate that $\mathrm{E}_{2}$-induced activation of the Erk1/2 pathway contributes to the survival advantage of ELT3 cells and 621-101 cells in detachment conditions. Since Bim is inactivated by proteasome-mediated degradation (26-28), we evaluated the effects of proteasome inhibition on Bim depletion and cell death signaling. We treated detached cells with bortezomib (BTZ) with concurrent $\mathrm{E}_{2}$ stimulation. FACS analysis revealed that BTZ blocked the $\mathrm{E}_{2}$-enhanced survival of ELT3 cells in vitro. (Figure 4A). The percentage of cells in late apoptosis (annexin $\mathrm{V}^{+} \mathrm{PI}^{+}$) was increased markedly in cells treated with BTZ plus $\mathrm{E}_{2}$ compared with that in cells treated with $\mathrm{E}_{2}$ alone (Figure $4 \mathrm{~B}$ ). Similarly, BTZ treatment restored the protein levels of Bim in $\mathrm{E}_{2}$-treated ELT3 cells (Figure 4C). Importantly, cells treated with $\mathrm{E}_{2}$ plus BTZ exhibited higher levels of cleaved caspase 3 and cleaved PARP compared with $\mathrm{E}_{2}$ treatment alone (Figure 4C). Similar findings were observed in detached 621-101 cells (Figure 4, D-F). Collectively, our data 


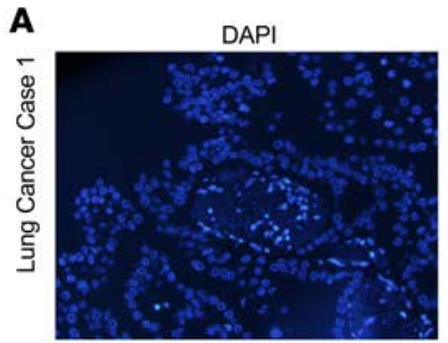

SMA
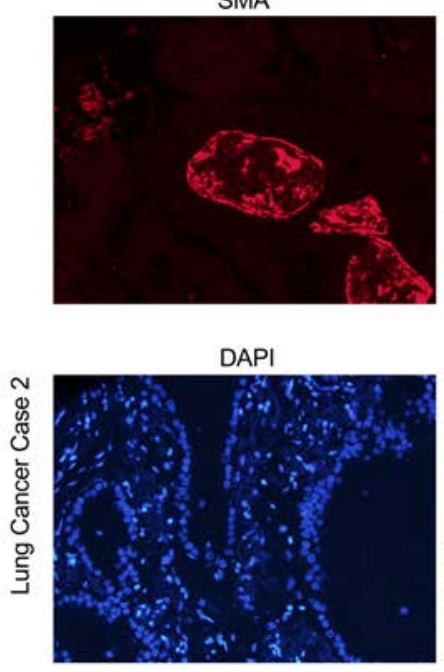

SMA

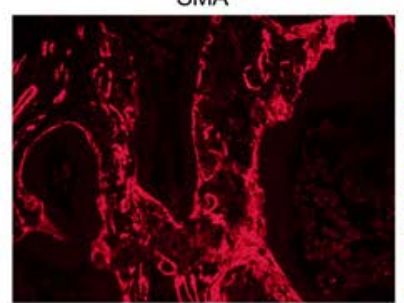

Bim

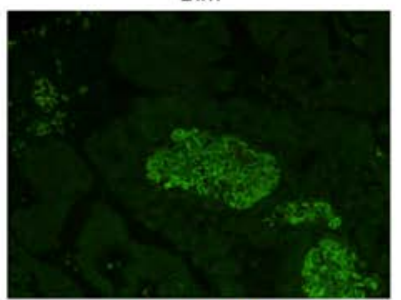

Bim/SMAVDAPI

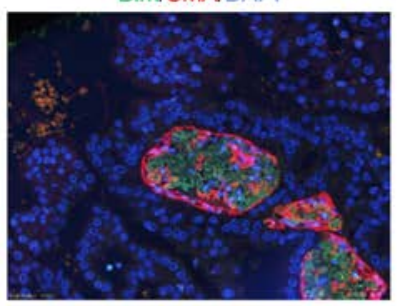

Bim

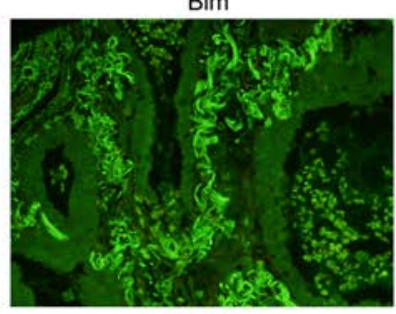

Bim/SMAVDAP|

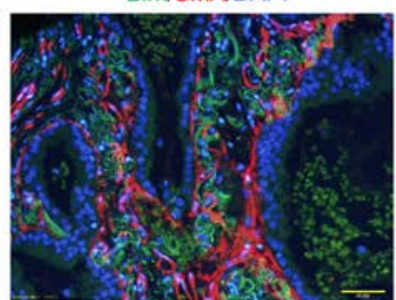

B

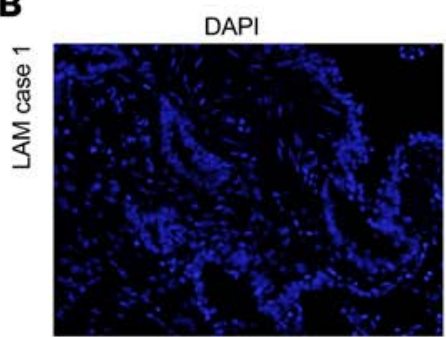

SMA

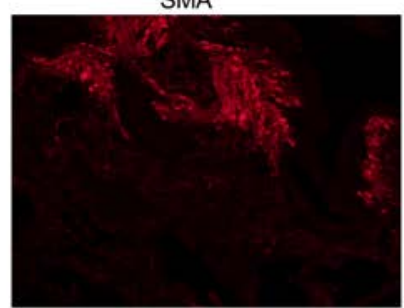

DAPI

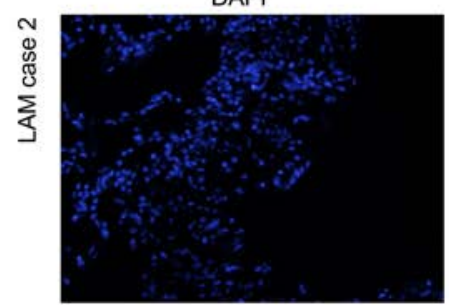

SMA

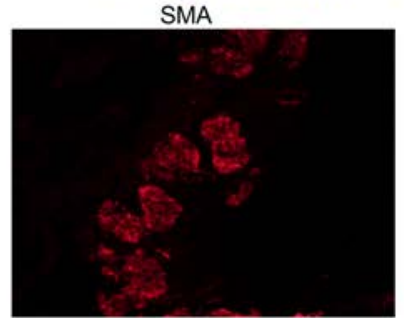

Bim

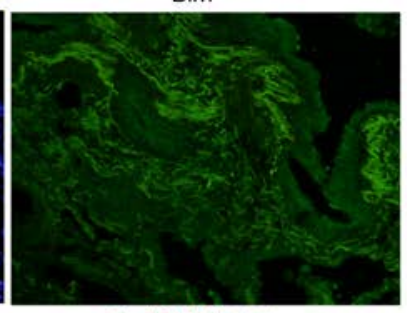

Bim/SMAVDAPI

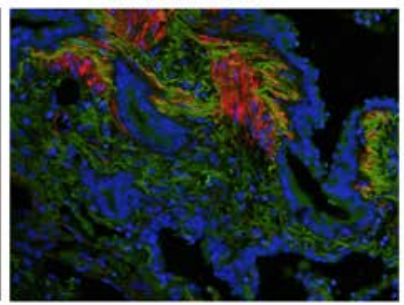

Bim

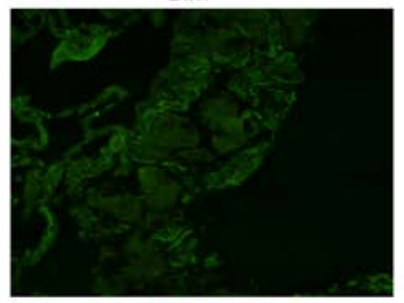

Bim/SMAVDAPI

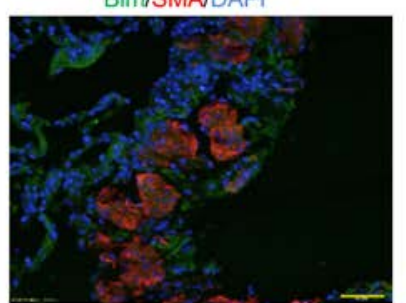

Figure 6. Low Bim accumulation is evident in LAM nodules. Immunofluorescence staining of Bim (green) and smooth muscle actin (SMA) (red) in 2 cases of (A) lung adenocarcinomas and 2 cases of (B) LAM lung tissues. Nuclei were stained with DAPI (blue). Scale bar: $50 \mu \mathrm{m}$.

support that Bim mediates $\mathrm{E}_{2}$-promoted resistance to anoikis in part via proteasome activity that can be targeted by BTZ in TSC2-deficient ELT3 and 621-101 cells in vitro. Of note, BTZ treatment attenuated $\mathrm{E}_{2}$-protected anoikis to a greater extent in ELT3 (Figure 4, A-C) than that in 621-101 cells (Figure 4, D-F), indicative of tissue specificity. Furthermore, we examined the effect of the combinatorial treatment of BTZ and PD98059 on the survival of ELT3 and 621-101 cells in the presence of $\mathrm{E}_{2}$ in detachment conditions. ELT3 cells treated with $\mathrm{E}_{2}$ plus BTZ and PD98059 exhibited higher levels of cleaved caspase 3 and cleaved PARP compared with $\mathrm{E}_{2}$ treatment alone (Figure 4C). Similar findings were observed in detached 621-101 cells (Figure 4, D-F), indicating that $\mathrm{E}_{2}$ protects cells from anoikis via multiple mechanisms.

$B T Z$ reverses $E_{2}$-promoted lung colonization of ELT3-luciferase cells in vivo. To further investigate the role of Bim in the survival of ELT3 cells in vivo, ERL4 cells cultured in detachment conditions were treated with $10 \mathrm{nM} \mathrm{E}_{2}$ or vehicle control for 24 hours. Cells were resuspended at the same density and intravenously injected into mice pretreated with BTZ 1 day prior to cell inoculation. At the baseline, similar levels of bioluminescence were observed in the chest regions of all mice (Figure 5A). At 6 and 24 hours after cell injection, the bioluminescence in the chest regions of mice inoculated with BTZ-treated cells was decreased by $40 \%$ and $70 \%$, respectively, compared with the controls (Figure 5, B and C). These data suggest that Bim plays a critical role in lung colonization by Tsc2-deficient ELT3 cells, in part owing to BTZ's ability to preserve Bim. 


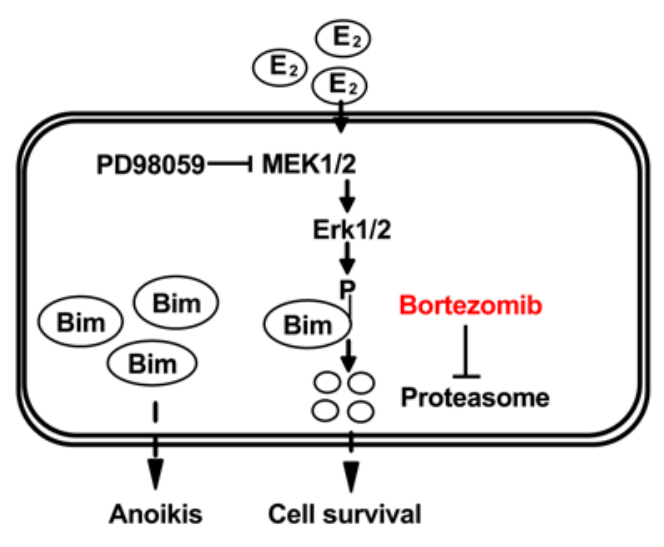

Figure 7. Hypothetical scheme of estrogen-enhanced survival of LAM patient-derived cells. Signaling event through estrogen $\left(E_{2}\right)$ stimulation results in: (i) MEK1/2-Erk1/2 cascades activation; (ii) phosphorylation and inactivation of the proapoptotic protein Bim; and (iii) proteasomal degradation of Bim, which protects cells against detachment-triggered cell death. Bortezomib inhibits proteasome activity toward protein degradation.

The expression of Bim is low in LAM lung nodule cells. It has been reported that Bim is overexpressed in lung adenocarcinomas relative to normal alveolus (29). We first validated the quality of Bim immunostaining using 2 cases of lung adenocarcinomas. As expected, Bim accumulation was distinct in nodular regions of both lung adenocarcinoma specimens compared with peripheral smooth muscle actin (SMA) immunoreactivity (Figure 6A). We then examined whether our in vitro and in vivo findings of Bim levels were relevant to human LAM. Immunofluorescence staining showed that SMA-positive LAM lesions express lower levels of Bim compared with adjacent lung parenchyma, whereas Bim accumulation is abundant in lung cells (Figure 6B).

\section{Discussion}

LAM is a predominantly female lung manifestation characterized by the accumulation of abnormal smooth muscle cells in the lung parenchyma and severe emphysema-like lung destruction that can lead to respiratory failure and mortality $(10,30-32)$. The marked gender specificity of LAM suggests that circulating female hormones including estrogen promote disease progression. We previously reported that estrogen promoted the survival of tuberin-deficient cells (9). In this study, we identified that Bim is a critical mediator of estrogen-enhanced survival in detachment conditions in LAM patient-derived cells. Importantly, targeting Bim turnover with proteasome inhibitors has a tumor suppression effect in animal models of LAM. Molecular and functional analyses of anoikis resistance of LAM patient-derived TSC2deficient cells may provide insights into the biology of LAM pathogenesis.

Adhesion to the appropriate extracellular matrix is necessary for normal stromal cells to survive and proliferate, and loss of this adhesion leads to anoikis. Anoikis prevents detached cells from reattaching to an inappropriate site and growing. Acquired resistance to anoikis allows tumor cells to survive and disseminate from the primary tumor site to form a distinct lesion at the distal site. Little is known about the molecular mechanisms underlying $\mathrm{E}_{2}$-induced survival and metastasis of tuberin-deficient cells. The impact of $E_{2}$ on the growth of tuberin-deficient cells has been reported $(9,33-40)$. Gu et al. reported that $\mathrm{E}_{2}$ activates Erk1/2 and increases the migration and invasion of tuberin-deficient cells (41). We hypothesize that $\mathrm{E}_{2}$ activates MEK1/2-Erk1/2 pathways to inhibit the accumulation and turnover of the proapoptotic protein Bim, leading to anoikis resistance in tuberin-deficient cells.

In this study, the MEK1/2 inhibitor PD98059, or the proteasome inhibitor BTZ, had a moderate effect on attenuation of $\mathrm{E}_{2}$-enhanced protection from apoptosis in detached TSC2-deficient cells. We also found that PD98059 treatment had limited inhibition of Erk1/2 activation in ELT3 cells and 621-101 cells, although $\mathrm{E}_{2}$-protected anoikis was not drastically attenuated by PD98059 treatment, suggesting that $\mathrm{E}_{2}$ functions through other mechanisms to suppress detachment-induced apoptotic machinery as reported by Frisch and Screaton (42). Moreover, BTZ treatment attenuated $\mathrm{E}_{2}$-protected anoikis to a greater extent in ELT3 than that in 621-101 cells, indicative of tissue specificity $(36,43,44)$. Importantly, the combinatorial treatment of PD98059 and BTZ induced more drastic anoikis in $\mathrm{E}_{2}$-treated ELT3 and 621-101 cells, further supporting the notion that $\mathrm{E}_{2}$ protects cells from anoikis via multiple mechanisms.

We previously found that $\mathrm{E}_{2}$ enhances the survival and metastasis of tuberin-deficient cells. In this study, we reinforce the concept that Bim is a key mediator of $\mathrm{E}_{2}$-promoted survival and we identify the signaling events that underlie the enhanced levels of Bim in $\mathrm{E}_{2}$-treated ELT3 cells. As a key BH3 protein regulating anoikis, Bim is regulated both transcriptionally and posttranslationally by the PI3K/Akt and Erk1/2 signaling networks (22). We provide evidence that a modest reduction in Bim expression can substantially increase the survival and metastasis of tuberin-deficient cells in vivo, and the proteasome inhibitor, BTZ, blocks the $\mathrm{E}_{2}$-induced survival of ELT3 cells in vivo (illustrated in Figure 7).

Little is known about the molecular mechanisms underlying LAM pathogenesis. Cells carrying TSC2 mutations have been identified in body fluids including blood, chylous effusions, and urine from 
women with LAM (11). We previously found that $\mathrm{E}_{2}$ enhanced the survival of disseminated ELT3 tumor cells and promoted the lung metastasis of ELT3 cells, both of which are associated with $\mathrm{E}_{2}$-induced anoikis resistance (9). LAM has been described as a destructive, low-grade metastasizing neoplasm (10). It is well known that in cancer cells including colon and mammary tumors, anoikis is triggered by detachment from the extracellular matrix and determined by Bim upregulation. Collectively, these findings imply that anoikis resistance may be a common mechanism leading to disease progression for LAM and other malignancies. Our data indicate that Bim accumulation and proteasome activity are critical components facilitating $\mathrm{E}_{2}$-enhanced survival in detachment conditions in LAM patient-derived cells. Thus, targeting Bim-mediated survival pathways including the proteasome may be a plausible future treatment strategy in patients with LAM.

\section{Methods}

Cell culture and reagents. ELT3 cells $(36,45)$ were provided by C. Walker, Institute of Biosciences and Technology, Texas A\&M University, Houston, Texas, USA. ERL4 (9) and LAM patient-associated angiomyolipoma-derived cells (621-101 cells) were provided by E.P. Henske, Brigham and Women's Hospital-Harvard Medical School $(9,22)$. Cells were cultured in DMEM/F12 supplemented with $10 \%$ FBS, $0.2 \mu \mathrm{M}$ hydrocortisone, $0.1 \mathrm{nM}$ triiodothyronine, $0.01 \mu \mathrm{U} / \mathrm{ml}$ vasopressin, $1.6 \mu \mathrm{M} \mathrm{FeSO}_{4}$, cholesterol, ITS, $100 \mathrm{ng} / \mathrm{ml} \mathrm{EGF,} 100 \mu \mathrm{g} / \mathrm{ml}$ zeomycin, and 1\% penicillin-streptomycin-amphotericin B. 17- $\beta$-estradiol ( $\mathrm{E}_{2}$ ) (10 nM, Sigma-Aldrich), PD98059 (50 $\mu \mathrm{M}$, Cell Signaling Technology), and BTZ (100 $\mathrm{nM}$, Velcade) were used as indicated.

Detached cell culture. Cells suspended in serum-free media were seeded onto 6-well plates (BD Falcon) coated with 1.2\% poly-HEMA (Sigma-Aldrich, P3932-10G), and treated with indicated reagents.

siRNA transfections. Two independent human Bim siRNAs (50 nM) (Dharmacon, LU-004383-00-0002 and L-004383-00-0005) were transfected into 621-101 cells, and 2 independent rat Bim-siRNAs (50 nM) (Dharmacon, LQ-093533-02-0002 and L-093533-02-0005) were transfected into ELT3 cells and ERL4 cells using Lipofectamine RNAiMAX (Invitrogen) according to the manufacturer's protocols. Cells were harvested 48 hours after transfection.

Quantitative RT-PCR. RNA from cultured cells was isolated using an RNeasy Mini Kit (Qiagen). Gene expression was quantified using One-Step qRT-PCR Kits (Invitrogen) in the Applied Biosystems Step One Plus Real-Time PCR System, and normalized to $\beta$-actin (human) or $\alpha$-tubulin (rat). Primers used for 621 101 cells and ELT3 cells were: human $\beta$-actin forward CACCATTGGCAATGAGCGGTTC and reverse AGGTCTTTGCGGATGTCCACGT; human Bim forward ATCGCCCTGTGGATGACTGAGT and reverse GCCAGGAGAAATCAAACAGAGGC; rat $\alpha$-tubulin forward GACCTGGAACCCACAGTTATT and reverse ATCTTCCTTGCCTGTGATGAG; rat Bim forward ATGGCCAAGCAACCTTCTGA and reverse GCTCCTGTCTTGCGATTCTG.

Immunoblotting and antibodies. Cells were lysed in m-PER buffer (Pierce). All primary antibodies were diluted in $1 \times$ phosphate buffered saline (PBS) supplemented with $0.5 \%$ Tween 20 (PBST). Antibodies used were Bim/BOD (diluted 1:500; ENZO, catalog ADI-AAP-330-E); $\beta$-actin (diluted 1:5,000; SigmaAldrich, catalog A5441); and the following from Cell Signalling Technology: phospho-Akt (S473) (diluted 1:1,000; catalog 9271); Akt (diluted 1:1,000; catalog 9272); phospho-Erk1/2 (T202/Y204) (diluted 1:2,000; catalog 9101); Erk1/2 (diluted 1:2,000; catalog 9102); phospho-S6 ribosomal protein (Ser235/236) (diluted 1:2,000; catalog 2211); S6 ribosomal protein (diluted 1:2,000; catalog 2317); cleaved caspase 3 (diluted 1:500; catalog 9661); cleaved PARP (human) (diluted 1:500; catalog 9541); cleaved PARP (rat) (diluted 1:500; catalog 9545).

Immunofluorescence staining. Sections were deparaffinized, incubated with anti-Bim $(1: 100$ in PBS $+3 \%$ BSA, ENZO, catalog ADI-AAP-330-E) and anti-SMA (1:200 in PBS + 3\% BSA, Santa Cruz Biotechnology, catalog sc32251), and secondary antibodies (1:1,000; Invitrogen, catalog A-21202 and A10042). Images were captured with an Olympus BX60 fluorescence microscope.

Fluorescence-activated cell sorting. An Annexin V: FITC Apoptosis Detection Kit I (BD catalog 556547) was used to stain detached ELT3 cells and 621-101 cells according to the manufacturer's protocols. FACS analysis was carried out on a BD FACSCanto II.

Animal studies. Intact or ovariectomized female C.B_Igh-1b/IcrTac-Prkdcscid (SCID) mice at 4 to 6 weeks of age were purchased from Taconic Biosciences. $\mathrm{E}_{2}$ was dissolved initially in $0.5 \mathrm{ml} 95 \%$ ethanol (stock concentration, $1 \mathrm{mM}$ ), and solubilized $\mathrm{E}_{2}$ was added to the drinking water to produce 
a concentration of $500 \mathrm{nM}$ for oral administration as previously described (46). The water intake was monitored and water bottles were changed twice per week. BTZ $(1 \mathrm{mg} / \mathrm{kg}$, dissolved in $100 \mu 1 \mathrm{l} \times \mathrm{PBS})$ was administered intraperitoneally $(47,48)$; the first dose was given 1 day prior to cell inoculation, and the second dose at 6 hours after cell inoculation. Cells $\left(2 \times 10^{5}\right)$ were injected into mice intravenously as previously described (9). Animal health was monitored daily during the tumor experiments. All mice were euthanized by carbon dioxide $\left(\mathrm{CO}_{2}\right)$ inhalation via compressed gas after the last image was taken.

Bioluminescent reporter imaging. Ten minutes prior to imaging, mice were given D-luciferin (120 mg/ kg, i.p., PerkinElmer Inc., catalog 122799). Bioluminescent signals were recorded using the Xenogen IVIS Spectrum System. Total photon flux of chest regions was analyzed as previously described (9).

Statistics. Data represent the mean \pm SEM. Statistical analyses were performed using a 2-tailed Student's $t$ test when comparing 2 groups for in vitro and in vivo studies, and 1-way ANOVA test (Dunnett's multiple comparisons test when comparing multiple groups with control group, Tukey's multiple comparisons test when making multiple pair-wise comparisons between different groups) for multiple group comparison. A $P$ value less than 0.05 was considered significant.

Study approval. The University of Cincinnati Standing Committees on Animals approved all procedures described according to standards as set forth in The Guide for the Care and Use of Laboratory Animals. The Institutional Review Board of the University of Cincinnati approved all relevant human studies.

\section{Author contributions}

CL, NL, XL, JS, TM, KM, YC, MJR, EPH, and JJY designed and performed in vitro studies. JL and JB designed and performed Bim knockdown in vitro experiments. CL and YS designed, performed, and analyzed in vivo experiments. XL and EYZ performed immunofluorescence staining. YZ provided guidance on cell survival experiments. JW and NAK examined lung adenocarcinoma sections. JJY contributed to the study design, implementation, and supervision of the study. CL and JJY wrote the manuscript. All authors had full access to the data, and approved the final version of the manuscript.

\section{Acknowledgments}

We are grateful to C. Walker (Texas A\&M Health Science Center) for providing ELT3 cells. We are also grateful to E. Kopras and F. McCormack for critical reading of the manuscript. J.L. was a Tuberous Sclerosis Alliance Postdoctoral Fellow. J.B. is a LAM Foundation Established Investigator. This study is supported in part by NIH grants HL098216 and DK098331, Department of Defense Exploratory Idea Development Award W81XWH-12-1-0442 (to J.J.Y.), The LAM Foundation Fellowship Award LAM00101F01-14 (to C.L.), and the Lucy J. Engles Fund for TSC and LAM Research (to E.P.H.).

Address correspondence to: Jane J. Yu, University of Cincinnati College of Medicine, Department of Internal Medicine, Pulmonary, Critical Care and Sleep Medicine, 231 Albert Sabin Way-ML 0564, Cincinnati, Ohio 45267, USA. Phone: 513.558.4114; E-mail: yuj9@ucmail.uc.edu.

1. Costello LC, Hartman TE, Ryu JH. High frequency of pulmonary lymphangioleiomyomatosis in women with tuberous sclerosis complex. Mayo Clin Proc. 2000;75(6):591-594.

2. Franz DN, et al. Mutational and radiographic analysis of pulmonary disease consistent with lymphangioleiomyomatosis and micronodular pneumocyte hyperplasia in women with tuberous sclerosis. Am J Respir Crit Care Med. 2001;164(4):661-668

3. Shepherd CW, Gomez MR, Lie JT, Crowson CS. Causes of death in patients with tuberous sclerosis. Mayo Clin Proc. 1991;66(8):792-796

4. Astrinidis A, et al. Mutational analysis of the tuberous sclerosis gene TSC2 in patients with pulmonary lymphangioleiomyomatosis. J Med Genet. 2000;37(1):55-57.

5. Kwiatkowski DJ. Animal models of lymphangioleiomyomatosis (LAM) and tuberous sclerosis complex (TSC). Lymphat Res Biol. 2010;8(1):51-57.

6. McCormack FX, et al. Efficacy and safety of sirolimus in lymphangioleiomyomatosis. N Engl J Med. 2011;364(17):1595-1606.

7. Karbowniczek M, et al. Recurrent lymphangiomyomatosis after transplantation: genetic analyses reveal a metastatic mechanism. Am J Respir Crit Care Med. 2003;167(7):976-982.

8. Logginidou H, Ao X, Russo I, Henske EP. Frequent estrogen and progesterone receptor immunoreactivity in renal angiomyolipomas from women with pulmonary lymphangioleiomyomatosis. Chest. 2000;117(1):25-30.

9. Yu JJ, et al. Estrogen promotes the survival and pulmonary metastasis of tuberin-null cells. Proc Natl Acad Sci U S A. 2009;106(8):2635-2640.

10. McCormack FX, Travis WD, Colby TV, Henske EP, Moss J. Lymphangioleiomyomatosis: calling it what it is: a low-grade, destructive, metastasizing neoplasm. Am J Respir Crit Care Med. 2012;186(12):1210-1212. 
11. Crooks DM, et al. Molecular and genetic analysis of disseminated neoplastic cells in lymphangioleiomyomatosis. Proc Natl Acad Sci U S A. 2004;101(50):17462-17467.

12. Akiyama T, Dass CR, Choong PF. Bim-targeted cancer therapy: a link between drug action and underlying molecular changes Mol Cancer Ther. 2009;8(12):3173-3180.

13. Balmanno K, Cook SJ. Tumour cell survival signalling by the ERK1/2 pathway. Cell Death Differ. 2009;16(3):368-377.

14. Noda T, Iwai S, Hamada M, Fujita Y, Yura Y. Induction of apoptosis of detached oral squamous cell carcinoma cells by safingol. Possible role of Bim, focal adhesion kinase and endonuclease G. Apoptosis. 2009;14(3):287-297.

15. Periyasamy-Thandavan S, et al. Insulin-like growth factor 1 attenuates antiestrogen- and antiprogestin-induced apoptosis in ER breast cancer cells by MEK1 regulation of the BH3-only pro-apoptotic protein Bim. Breast Cancer Res. 2012;14(2):R52.

16. Delgado M, Tesfaigzi Y. BH3-only proteins, Bmf and Bim, in autophagy. Cell Cycle. 2013;12(22):3453-3454.

17. Tan N, et al. Bcl-2/Bcl-xL inhibition increases the efficacy of MEK inhibition alone and in combination with PI3 kinase inhibition in lung and pancreatic tumor models. Mol Cancer Ther. 2013;12(6):853-864.

18. Zhang LN, Li JY, Xu W. A review of the role of Puma, Noxa and Bim in the tumorigenesis, therapy and drug resistance of chronic lymphocytic leukemia. Cancer Gene Ther. 2013;20(1):1-7.

19. Kumasaka T, et al. Lymphangiogenesis-mediated shedding of LAM cell clusters as a mechanism for dissemination in lymphangioleiomyomatosis. Am J Surg Pathol. 2005;29(10):1356-1366.

20. Carsillo T, Astrinidis A, Henske EP. Mutations in the tuberous sclerosis complex gene TSC2 are a cause of sporadic pulmonary lymphangioleiomyomatosis. Proc Natl Acad Sci U S A. 2000;97(11):6085-6090.

21. Panka DJ, Atkins MB, Mier JW. Targeting the mitogen-activated protein kinase pathway in the treatment of malignant melanoma. Clin Cancer Res. 2006;12(7 Pt 2):2371s-2375s.

22. Hong F, Larrea MD, Doughty C, Kwiatkowski DJ, Squillace R, Slingerland JM. mTOR-raptor binds and activates SGK1 to regulate p27 phosphorylation. Mol Cell. 2008;30(6):701-711

23. Krymskaya VP, Goncharova EA. PI3K/mTORC1 activation in hamartoma syndromes: therapeutic prospects. Cell Cycle. 2009;8(3):403-413.

24. Paraiso KH, et al. PTEN loss confers BRAF inhibitor resistance to melanoma cells through the suppression of BIM expression. Cancer Res. 2011;71(7):2750-2760

25. Barnes EA, Kenerson HL, Jiang X, Yeung RS. Tuberin regulates E-cadherin localization: implications in epithelialmesenchymal transition. Am J Pathol. 2010;177(4):1765-1778.

26. Collins NL, Reginato MJ, Paulus JK, Sgroi DC, Labaer J, Brugge JS. G1/S cell cycle arrest provides anoikis resistance through Erk-mediated Bim suppression. Mol Cell Biol. 2005;25(12):5282-5291.

27. Quadros MR, Connelly S, Kari C, Abrams MT, Wickstrom E, Rodeck U. EGFR-dependent downregulation of Bim in epithelia cells requires MAPK and PKC-delta activities. Cancer Biol Ther. 2006;5(5):498-504.

28. Reginato MJ, et al. Integrins and EGFR coordinately regulate the pro-apoptotic protein Bim to prevent anoikis. Nat Cell Biol. 2003;5(8):733-740.

29. Sakakibara-Konishi J, et al. Expression of Bim, Noxa, and Puma in non-small cell lung cancer. BMC Cancer. 2012;12:286.

30. Henske EP, McCormack FX. Lymphangioleiomyomatosis - a wolf in sheep's clothing. J Clin Invest. 2012;122(11):3807-3816.

31. Krymskaya VP. Treatment option(s) for pulmonary lymphangioleiomyomatosis: progress and current challenges. Am J Respir Cell Mol Biol. 2012;46(5):563-565.

32. Taveira-DaSilva AM, Pacheco-Rodriguez G, Moss J. The natural history of lymphangioleiomyomatosis: markers of severity, rate of progression and prognosis. Lymphat Res Biol. 2010;8(1):9-19.

33. El-Hashemite N, Walker V, Kwiatkowski DJ. Estrogen enhances whereas tamoxifen retards development of Tsc mouse liver hemangioma: a tumor related to renal angiomyolipoma and pulmonary lymphangioleiomyomatosis. Cancer Res. 2005;65(6):2474-2481

34. Finlay GA, Thannickal VJ, Fanburg BL, Kwiatkowski DJ. Platelet-derived growth factor-induced p42/44 mitogen-activated protein kinase activation and cellular growth is mediated by reactive oxygen species in the absence of TSC2/tuberin. Cancer Res. 2005;65(23):10881-10890.

35. Finlay GA, et al. Estrogen-induced smooth muscle cell growth is regulated by tuberin and associated with altered activation of platelet-derived growth factor receptor-beta and ERK-1/2. J Biol Chem. 2004;279(22):23114-23122.

36. Howe SR, Gottardis MM, Everitt JI, Walker C. Estrogen stimulation and tamoxifen inhibition of leiomyoma cell growth in vitro and in vivo. Endocrinology. 1995;136(11):4996-5003.

37. Li C, et al. Estradiol and mTORC2 cooperate to enhance prostaglandin biosynthesis and tumorigenesis in TSC2-deficient LAM cells. J Exp Med. 2014;211(1):15-28.

38. Li C, et al. Faslodex inhibits estradiol-induced extracellular matrix dynamics and lung metastasis in a model of lymphangioleiomyomatosis. Am J Respir Cell Mol Biol. 2013;49(1):135-142.

39. Liu F, et al. Real-time monitoring of tumorigenesis, dissemination, \& drug response in a preclinical model of lymphangioleiomyomatosis/tuberous sclerosis complex. PLoS ONE. 2012;7(6):e38589.

40. Sun Y, et al. Estradiol promotes pentose phosphate pathway addiction and cell survival via reactivation of Akt in mTORC1 hyperactive cells. Cell Death Dis. 2014;5:e1231.

41. Gu X, Yu JJ, Ilter D, Blenis N, Henske EP, Blenis J. Integration of mTOR and estrogen-ERK2 signaling in lymphangioleiomyomatosis pathogenesis. Proc Natl Acad Sci USA. 2013;110(37):14960-14965.

42. Frisch SM, Screaton RA. Anoikis mechanisms. Curr Opin Cell Biol. 2001;13(5):555-562.

43. Howe SR, Gottardis MM, Everitt JI, Goldsworthy TL, Wolf DC, Walker C. Rodent model of reproductive tract leiomyomata. Establishment and characterization of tumor-derived cell lines. Am J Pathol. 1995;146(6):1568-1579.

44. Yu J, Astrinidis A, Howard S, Henske EP. Estradiol and tamoxifen stimulate LAM-associated angiomyolipoma cell growth and activate both genomic and nongenomic signaling pathways. Am J Physiol Lung Cell Mol Physiol. 2004;286(4):L694-L700.

45. Howe SR, Gottardis MM, Everitt JI, Goldsworthy TL, Wolf DC, Walker C. Rodent model of reproductive tract leiomyomata. Establishment and characterization of tumor-derived cell lines. Am J Pathol. 1995;146(6):1568-1579.

46. Gordon MN, Osterburg HH, May PC, Finch CE. Effective oral administration of 17 beta-estradiol to female C57BL/6J mice 
through the drinking water. Biol Reprod. 1986;35(5):1088-1095.

47. Wagner-Ballon $\mathrm{O}$, et al. Proteasome inhibitor bortezomib impairs both myelofibrosis and osteosclerosis induced by high thrombopoietin levels in mice. Blood. 2007;110(1):345-353.

48. Sarosiek KA, et al. Efficacy of bortezomib in a direct xenograft model of primary effusion lymphoma. Proc Natl Acad Sci U S A. 2010;107(29):13069-13074. 